\title{
A model of three-way decisions for Knowledge Harnessing
}

\author{
Gonzalo A. Aranda-Corral a, Joaquín Borrego-Díaz ${ }^{\text {b,* }}$, Juan Galán-Páez ${ }^{\text {b,c }}$ \\ a Department of Information Technology, Universidad de Huelva, Crta. Palos de La Frontera s/n. 21819 Palos de La Frontera, Spain
b Departamento de Ciencias de la Computación e Inteligencia Artificial, E.T.S. Ingeniería Informática, Universidad de Sevilla, Avda. Reina Mercedes s.n. 41012- \\ Sevilla, Spain \\ c Datrik Intelligence S.A., Spain
}

Keywords:

Three-way decisions

Knowledge-based systems

Variable forgetting

Knowledge Harnessing

\begin{abstract}
A B S T R A C T
The present work introduces the Knowledge Harnessing, by showing its theoretical foundations as well as a three-way decision model to deal with it. The problem poses how to extract valid information about a specific context from conflicting or uncertain information received by a system (or agent). With this aim, forgetting variable operators are used to both characterize the problem from the logical point of view and provide a theoretical solution as an acceptance-rejection problem. Since the formalization is semantic in nature (it considers the models of the knowledge base that admit the extracted knowledge), general bounds are provided for acceptance-rejection evaluation on boundary region.
\end{abstract}

\section{Introduction}

The task of designing deliberative agents implies solving the challenge of defining the behavior of the agent based on the logical consequence from its knowledge base (or beliefs) or variants thereof. The problem of logical entailment is to decide whether a given formula $F$ is valid in all models of a Knowledge Base (KB) $\mathrm{K}(K \models F)$.

In some cases it is possible that $K \not \models F$ and $K \not \models \neg F$ occurs, and therefore it is not possible to decide, by entailment, on the information represented by $F$, since in this case both $K \cup\{F\}$ and $K \cup\{\neg F\}$ would be consistent. In this situation, a classic alternative in AI is to design other methods based on non-classical logic (e.g. with non-monotonous reasoning [1]). Often, methods are designed for the case of syntactically simple formulas (being $F$ an atom or clause). If $F$ represents complex information (with a syntactic complex structure), the engineer have to design methods to take advantage (by some appropriate procedure that does not cause inconsistency) of some information contained in $F$ on which he/she can decide its validity in order to deal with it.

This paper is focused on a way of solving this kind of issues. The aim of this work is to explain how to use conservative retraction methods to formalize a Three-Way Decision (TWD) model for dealing with what we have called the Knowledge Harnessing problem. This is introduced in this paper for the first time. The problem, referred to Knowledge-Based Systems (KBS), consists in retrieving useful knowledge that is implicitly contained in some information received by an agent/system, when it is either incompatible with its knowledge (causes inconsistency) or simply it is not logical consequence from it. Formally, for context of this article, it will be limited to harnessing nontrivial knowledge contained in the conflicting information that refers to certain sets of variables (usually variable sets that determine the context in which the agent is working). A way of extracting such kind of knowledge is based on distilling the information to obtain only the relationships

\footnotetext{
* Corresponding author.

E-mail address: jborrego@us.es (J. Borrego-Díaz).
} 


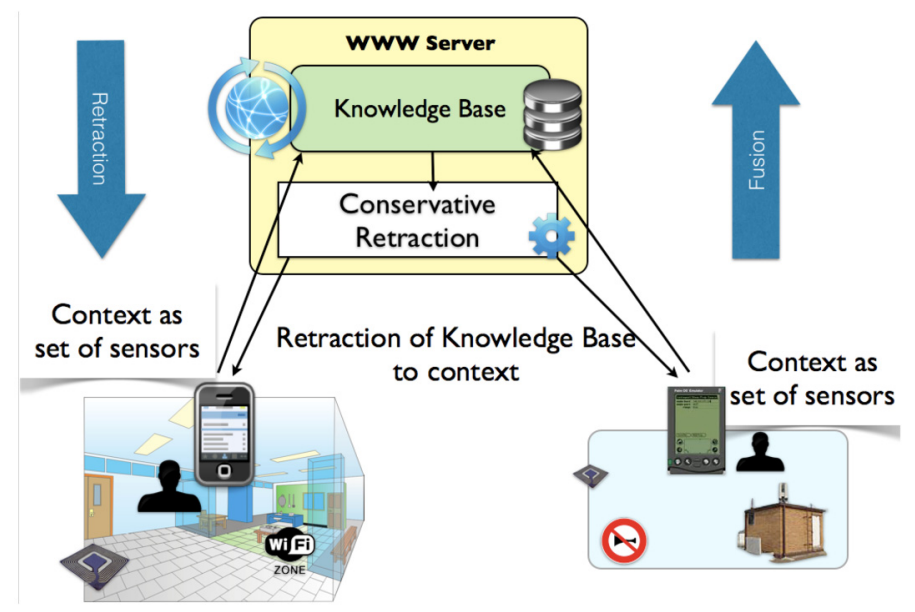

Fig. 1. Conservative retraction as a mobile service for mobile agents (top-down process) in contraposition to knowledge fusion as a bottom-up process.

between the variables that are useful for our purposes, and forgetting the rest. The variable forgetting problem [2] has received considerable attention within the logic-based AI research field. The aim is to remove any reference to the variable to be forgotten from the KB but keeping the information that concerns the rest of them.

\subsection{Variable forgetting}

Variable forgetting (firstly introduced in [3]) is a type of reduction on a KB, $K$, that eliminates all information about the forgotten variable by getting another $\mathrm{KB}, K^{\prime}$, which retains all the knowledge that represents $K$ over the rest. For such reduction to be successful, $K$ must be a conservative extension of $K^{\prime}$ (or, equivalent, $K^{\prime}$ a conservative retraction of $K$ ). In general terms, a knowledge base of $K^{\prime}$ in the language $\mathcal{L}^{\prime}$ is a conservative retraction of another $K$ if $K$ is an extension of $K^{\prime}$ but all $\mathcal{L}^{\prime}$-formula that entails $K$ is also entailed by $K^{\prime}$. That is to say, the conservative retraction $K^{\prime}$ restricts the knowledge to the language $\mathcal{L}^{\prime}$ but without losing deductive power in that language. Therefore, all the knowledge of that type of $K$ is preserved. Variable forgetting is simply a conservative retraction that removes only one variable from the language.

Variable forgetting is a technique widely studied in AI. Applications have been developed in the field of programs refinement or update (Logic [4], rule-based [5] and Answer Set Programs [6]), in the field of Constraint Satisfaction Problems [7] as well as in non-classical logic (see e.g. [8,9]).

\subsection{Motivating example: top-down processes for agents within mobile devices versus for knowledge harness as services}

A framework in which the use of Knowledge Harnessing would be very inspiring is the domain of context-based reasoning. It is usual for a context to be specified by the set of variables whose values characterize it (these values represent the information the context contains in a specific state). The main problem is to specialize the global KB to the specific context in which the agent/system is working, by limiting the KB to knowledge about the variables that represent it.

The use of conservative retraction in a multi-agent mobile infrastructure (MAS) has been chosen as example. This case study motivated the analysis of the problem (that came up in [10] and [11]) presented in this paper. Designers have mainly two natural options (see Fig. 1). The first is the implementation of agents inside mobile devices. This option is conditioned by the heterogeneity of mobile devices (both software and hardware). Additionally, if the designer wants to combine results from different devices, other methods (knowledge/belief merging, Fig. 1, left) are required. These operations would involve the fusion of knowledge from different contexts, often determined by sensors activated in the environment. The second option consists in implementing the agents within a MAS in the Cloud, providing (through the mobile Internet network) the services that mobile users could require. The second option seems more feasible than the first since agents live in a pre-set MAS and therefore, problems related to device heterogeneity disappear. Applications on the mobile device simply have to provide communication between the agents within the MAS and the devices (for example). However, in this case, the traffic between mobile devices and the MAS would be higher than in the first option. Furthermore, in a centralized MAS there is no initial adaptation of the mobile device to the context in which the user finds himself (it must be taken into account that, potentially, the device can be in an excessive number of contexts). Thus, a top-down procedure is required to provide specialized knowledge to the mobile device, possibly specialized in the context (set of sensors/signals active or available). It could therefore happen that even in MAS, agents have different KBs; in fact it will be advisable if they are going to serve as an inference mechanism for the mobile device.

There is an intermediate option that can solve the use of different knowledge bases by agents. The idea consists in keeping an unique global knowledge base and implement a service able to provide complete information about the context (associated to the sensors/signals) in which each mobile agent is working. Such information could be extracted from the 
global knowledge base KB by using conservative retraction (Fig. 1, right). The advantage of this option is that it does not need a method to combine different knowledge bases (as it happened in the first option), and the designer only has to build one global knowledge base (whereas in the second option it was possible for different agents to handle different knowledge bases without having to manage the global knowledge base). Finally, another advantage of this option is that the conservative retraction operation can be implemented as a service of the MAS and not as a program of each agent.

Note that the knowledge in the language associated with the context has not been weakened so far. That is to say, by the conservative retraction definition, the original KB and the sub-KB built are equivalent (on the sublanguage). Hopefully the sub-KB is simpler than the global $\mathrm{Kb}$ and therefore its processing will be less costly.

Going deeper into the example, it would be necessary to establish a framework to study situations in which the specialized KB of the mobile device (we are going to suppose that it is the conservative retraction of the general knowledge to the variables of the concrete context) is not able to decide about external information (for example sensors or data introduced by the user) because some kind of conflict appears. That is to say, a possible case in which the decision on the validity of the information cannot be decided by the KB. This is the case we would deal with by using Knowledge Harnessing to extract valid information from the conflictive one (inconsistent, incoherent, invalid, with uncertainty, etc.). It could be interesting to provide that framework with a three-way decision model to work with this kind of problems. Note that, since the conservative retraction is equivalent (in the reduced language) to the global $\mathrm{KB}$, solving this problem in the mobile device's specialized KB is equivalent to doing so in the global KB in case it was necessary. Thus, it is interesting to apply Knowledge Harnessing methods to the former.

\subsection{Deliberative agents, conflicting information and Knowledge Harnessing}

The reliable information that can be extracted from the conflicting information that has been received would be very useful for several types of agent-based systems since it would provide information with which the agent could work in deliberative knowledge-based agents, as for example, those based on GOLOG [12] and particularly in its IndiGOLOG extension [13], which allows the incremental implementation of plans taking into account the assimilation of external information coming from the detection and monitoring of events. In general terms, in other models with mental categories this would allow the designer to modify the behavior scheme of the agent extending the theory that supports the model to use the knowledge harnessed. For instance, in models in which the agent is supposed to be skeptical and maintain a persistent belief base unless he or she perceives contradictions.

In more limited models of agency of reactive nature such as the Teleo-reactive one (TR) [14], the knowledge harnessing from the agent/robot perceptions would be very useful for the designer, since it could refine the TR succession defined by regression that specifies the behavior of the agent (it would allow him to reorder this succession to face this type of cases in the future).

In the context of multiagent knowledge processing and retrieval, the negotiation strategies between agents to reconcile knowledge $[15,16]$ would allow the agent who receives information from the other agent to assess how conflicting it is with respect to his knowledge, so that he can evaluate his acceptance and proceed to conciliate with the other agent.

\subsection{Knowledge Harnessing}

The present paper introduces what we have called the Knowledge Harnessing problem. Roughly speaking, it poses how to extract, from conflictive information, knowledge that an agent can entail as true.

Given $K$ a knowledge base, $\mathcal{L}_{0}$ a language sub-language of $K$, and $F$ the formula representing the information received, it is said that there is knowledge harnessed from $F$ with respect to $\mathrm{K}$ and $\mathcal{L}_{0}$ (denoted by $K \models_{\mathcal{L}_{0}} F$ ) if the conservative retraction of $F$ to $\mathcal{L}_{0}$, denoted by $F_{\left\lceil\mathcal{L}_{0}\right.}$, is not trivial (i.e. it provides non-trivial information, that is, it is not a tautology) and it is entailed by $K$, that is, $K \models F_{\mid \mathcal{L}_{0}}$. The idea behind the definition is that if we specialize $F$ to the context determined by the sublanguage $\mathcal{L}_{0}$, then we can certify its validity, although the full information represented by $F$ does not. Knowledge harnessing is important because it represents a way to extract useful knowledge in situations where all the information available is uncertain, contradictory or undecidable from agent's knowledge. The problem definition itself shows that the use of forgetting operators significantly helps its resolution

From the same definition it is intuited that the availability and handling of operators to forget variables makes it easier for solving the questions.

When the knowledge to be harnessed is not unique, formal solutions to assess the different alternatives are necessary, thus it is worthy using a TWD schema to modelize these problems. As simple example could be

$$
K=\{p \leftrightarrow q \vee r\} \text { and } F=\{p \wedge \neg q\}
$$

This is an extreme case where a TWD approach is interesting since $K \not \forall F$ and none of the retractions of $F$ to sublanguages of $K,\{p, q, r\}$, provide a solution. These retractions are (calculations are not detailed)

$$
F_{\lceil\{p, q, r\}}=F, F_{\lceil\{p\}}=p, F_{\lceil\{q\}}=\neg q, F_{\lceil\{r\}}=\top
$$

and only the last one is logical consequence of $K$. However, it is not informative, that is to say, it is not a formula providing specific information since it is a tautology. The other three retractions are informative when values for the variables are 
provided but they are not logical consequence of $k$. Therefore, it is necessary to assess their suitability in some way. This paper presents a semantic approach to measure such suitability. This approach measures how conservative is each option, that is to say, how many models of $K$ satisfy each formula (the proportion of models). In the example, there exist: only one model satisfying $F_{\lceil\{p, q, r\}}$, three models of $K$ satisfying $F_{\lceil\{p\}}$ and only two satisfy $F_{\{\{q\}}$. Therefore, the second would be chosen. This option implies selecting which informative sublanguages are the most suitable in each case, by comparing the associated measures.

It is important to note that Knowledge Harnessing is not a belief revision variant (particularly updating) [17]. The goal of the later is to modify the belief base of the agent in order to accept the new information in a consistent way. That is to say, $K$ would be transformed and $F$ would be accepted as valid. In the Knowledge Harnessing $F$ is transformed so the information can be accepted by $K$.

The approach is based in modifying the information in order to obtain a formula, in the same language than the initial KB so it is possible to check whether it is logical consequence. Therefore it is not advisable to use a representation logic (e.g. trivalued propositional logic) different than that of the initial base.

\subsubsection{Notions related to Knowledge Harnessing}

As mentioned above, the notion of Knowledge Harnessing is introduced for the first time in this document. As far as the authors know, it has not been discussed in general (not even for the logic on which this paper is focused, propositional logic). However, there are concepts and techniques that are applied in several fields that could be considered as specific cases or variants of Knowledge Harvesting

As it was already mentioned, the reason why the new information $F$ is in conflict with the $\mathrm{KB}, K$, consist of many causes, among others: inconsistency, the fact that it is not valid information in the agent's work context, the fact that it is inconsistent from the agent's designer point of view, and finally, that it is affected by uncertainty. Let us analyze some works that deal with these problems and their relation with the notion of $\mathrm{KH}$.

Perhaps the most studied case is that of the inconsistency of the KB, which although it is not our case (we work with consistent KB) some of the solutions offered can be applied as particular cases of KH. In the case of propositional logic (and in general all logic understood in Tarski's sense, that is, using the operator that determines all the consequences), the mechanism of choosing options [18] to obtain consistent sub-KBs from the inconsistent set $K+\{F\}$ can be used to consider only options that entails $K$. If $K$ (or database) is inconsistent, then Knowledge Harnessing would not be applied (it doesn't make sense since $K$ would imply any formula), although some of the techniques used to be able to work with queries on inconsistent databases [19] are related to the KH. For example, ontology repair techniques (in the sense of [20]) can be considered as a variant of $\mathrm{KH}$ for the case in which the $\mathrm{KB}$ (the ontology) is not valid (it does not have as a model the universe to be represented even if it is consistent).

Likewise, conflicts between two ontologies are located in axioms that are susceptible to be repaired. For instance, assuming that one ontology is correct and the problem of conflict comes from a second one. From the point of view of $\mathrm{KH}$, a disagreement between a $\mathcal{O}_{1}$ ontology with $F$, a result deduced from another $\mathcal{O}_{2}$, can be repaired by using argumentative techniques [21]. This technique is an opportunity, since it reduces the problem to repairing the conflict of some of the axioms of the second one that are used in the argument that entails $F$. In the case of DL-ontologies, this form of argumentbased handling of the disagreements involves, in fact, knowledge harnessed from axioms of $\mathcal{O}_{2}$. The same idea is valid for inconsistency conflicts in Peer-to-Peer systems [22].

Another case that we could consider as a variant of the Knowledge Harnessing problem is that of enriching an ontology with information (or concepts) mined from data sources (e.g. to populate the ontology [23]). In this case the information obtained, being subject to Natural Language Processing, can be conflicting and must be refined, possibly restricting its expressiveness, in order to be added to the ontology.

In the field of databases, the option to modify (repair) the query (because it enters into some kind of conflict with the database) to obtain an approximate answer is a very similar problem. The case where the set of answers to a query is empty (the so-called no solution could be found dilemma) is important in scenarios such as recommendation systems because of its impact on e-commerce [24,25]. Among the different possible solutions, one is to adapt the query to validate some kind of approximate response, including the use of different database sources [26], in cloud computing scenarios [27] or by means of mediators [28]. For that dilemma, $\mathrm{KH}$ can provide weak versions of the query that are extremely interesting since we would get an answer. In the case of e-commerce, the customer who made the query receives a recommended product similar to the one he/she is looking for, consistent with the database and therefore with the current stock of the platform.

Abductive reasoning can be used to extract information compatible with the (rule-based) KB [29] from a query. This option does not allow obtaining information already contained in $F$, and it would be in principle more general. In [30] the generalization is applied to the particular case of conjunctive queries, which would correspond to a special case of $\mathrm{KH}$ when $F$ is a conjunction of literals.

\subsection{Three-way decisions}

The concept of TWD has recently been proposed in order to study the decision-making processes in a general way (see e.g. [31,32]), unifying in the same framework different approaches to decision making that deal with the case of indefinition 
or uncertainty (that is, approaches in which acceptance, non-commitment, and rejection are considered) such as the field of Granular Computing [33]. Such approach is natural from a cognitive point of view [34] and therefore very interesting for its use in the design of representation and reasoning processes in models of agents to deal with the rules that handle indecision.

The basic idea guiding TWD is to work on the problem of interpreting the workspace domain (system inputs, beliefs, etc.) by approaching it with three regions intimately related to decision-making procedures of any nature (see [35] for a introduction). More specifically, three regions, the positive, negative and border ones, are considered from the outset to be the regions of acceptance, rejection and non commitment in a ternary classification, respectively. Naturally, positive and negative regions could induce the rules of acceptance and rejection whilst the border region represents the no decision (or direct commitment) about its possible acceptance. The idea is very inspiring because when delimiting the region of indecision, multitude of techniques and a good number of approximations for rough or soft reasoning could be applied.

Since TWD aims to encompass processes in the presence of three decision situations, there are multiple ways of formalizing the idea (both those recovered from the same fields that are intended to be unified and those new ones inspired by the new paradigm). They reflect different ways of understanding both the decision process and the ontological nature of the positive, negative and borderline sets (an excellent exposition of the different approaches is again provided by [35]): evaluation on posets and decision determination, to work with interval-based evaluations, rough set theory, multi-valued logics and extensions of Formal Concept Analysis (FCA), among others.

With respect to the field this paper deals with (the Knowledge Level AI viewpoint), there are well known techniques addressing the problem of non-commitment (the third case besides acceptance and rejection) that can be seen as a solution within AI modeling (for example, the Closed World Assumption, where the non-entailed atoms are classified as rejected). In the field of agent theory, where the problem of undecidability (from the agent's knowledge point of view) about the action to be taken is ubiquitous, the treatment of its beliefs (and knowledge) about the world, and the analysis of the possible situations, requires solving such indecision. Thus, decision making within the non-commitment region is a major concern for the deliberative agent models cited above.

\subsection{Aim and structure of the paper}

The aim of this paper is to provide the formal foundations of a TWD model for Knowledge Harnessing. We are mainly interested in providing tools to take decisions on boundary regions for the classical (bivalued) logic. This paper is focused on propositional logic where some semantic features (number of models of a KB, for example) can be computed and thus used -at least theoretically- to estimate decisions and rejections.

The content of the paper is structured as follows. The following section is devoted to introduce the three main elements that are combined in the article (variable forgetting, Knowledge Harnessing and TWD theory). Its goal is twofold, to unify notation and to provide the formal elements used in following sections. In Sect. 3 the notion of Knowledge Harnessing is formalized and basics properties are proved. From this formalization the TWD model is introduced and studied in Sect. 4. Finally, some illustrative examples are presented in Sect. 5 and conclusions and future work in Sect. 6.

\section{Background}

Throughout the paper it is assumed that the reader is familiar with propositional logic, although a section about basic elements and notation used in the paper is introduced.

\subsection{Evaluation-based three-way decision model}

As commented in the introduction, the TWD is not limited to the study and design of those decision regions since the partitioning must involve corresponding decision processes that lead the agent/system to act. According to Y. Yao [34], the processes of trisecting the universe of discourse (which involve tasks such as trisecting in three regions, designing functions for evaluating acceptance/rejection or finding thresholds determining the regions) must be supplemented by other activities. Namely the resolution of problems associated with the action to be taken, such as the appropriate description of each region in order to identify the region a particular situation belongs or will belong to, the transfer of an object between regions according to the interests of the agent, and the design of behavioral strategies in each of these regions.

In addition to the two research activities mentioned above, a third one should be added [33], that is focused on how to evaluate the effectiveness of the designed strategy.

This section is focused on a specific framework for TWD, among the existing ones [35]. In this framework, TWD is represented through an evaluation that classifies entries as accepted, rejected, or unknown. The general formulation suggests working with evaluations with rank on a poset. For this particular case, the range of values of the evaluation are the (ordered) interval $[0,1]$, identifying 0 with false and 1 with true. An acceptance-rejection evaluation is a function

$$
\omega: U \rightarrow[0,1]
$$

and the three decision regions are 
- $P O S_{\omega}=\{u \in U: \omega(u)=1\}$

- $N E G_{\omega}=\{u \in U: \omega(u)=0\}$

- $B N D_{\omega}=\{u \in U: 0<\omega(u)<1\}$

No more elements of this approach will be detailed here (we refer the reader to [35]), since these will be introduced when we study the specific approach of the paper.

In our case we intend to estimate how close is the information represented by $F$ from the knowledge represented by the knowledge base $K$, from a semantic point of view (considering models of $K$ ). To a certain extent the evaluation can be seen as a degree of certainty similar to fuzzy logic, by taking into account as degree of truth the value of $\omega$ that tells us for how many models of $K$ the information really has harnessed knowledge. The notions of logic that will be used are introduced next.

As mentioned above, the TWD considers all aspects of decision making, not just the analysis of the three general decision options such as that based on an assessment of the three regions defined above. Note that these are closely linked to the other tasks complementing the decision making process itself (data collection/indexes, analysis of previous decision making, justification/explaining, etc.). In fact, as it can be seen from the bibliography in this paper, these complementary tasks can also be studied from the perspective of the TWD in order to prepare or improve decision making processes.

Within the decision making process, there are different solutions depending on both the ontological nature we consider for those three alternatives and the different alternatives offered by TWD: three sets of options, through functions that estimate the goodness of each possible decision (the extremes being acceptance-rejection), fuzzy sets, etc. The selection depends on the nature of the problem and the designer's options. In this way, it is possible to apply the principles of TWD in fields such as Data Science (included Big Data [36]) and Artificial Intelligence, where the applications range from the foundation of Machine Learning processes that use rough techniques providing solutions to be applied in the presence of uncertainty or absence of data/information [37], dealing with imbalanced data [38], ambiguity in labeling data [39] or the need for incremental concept learning [40], to more challenging applied fields such as the analysis of feelings and opinions $[41,42]$.

In the case under discussion in this paper (the use of TWDs to resolve problems with conflicting information received by the agent), the effectiveness (validity) and correctness of the solution must be considered at two levels. The first is the logical one, namely the correctness of the proposed solution (which is discussed in this paper, mainly in section 4). Solving this problem is mandatory if one wishes to address the second, which is the (experimental) validity of the solution (that in this case is outside the scope of this paper and depends on the theory of the chosen agency) in cases of application.

\subsection{Conservative retraction in propositional logic}

The purpose of this section is to briefly recall the main concepts of propositional logic, as well as to specify the notation used along the paper. A propositional language is a set $\mathcal{L}=\left\{p_{1}, \ldots, p_{n}\right\}$ of propositional symbols (variables). The set of formulas $\operatorname{Form}(\mathcal{L})$ is built up in the usual way, by using the connectives $\neg, \wedge, \vee, \rightarrow$ and $\top$ ( $\top$ denotes true, and $\perp$ is $\neg \top$ ).

An interpretation (or valuation) $v$ is a truth value assignation $v: \mathcal{L} \rightarrow\{0,1\}$. It is said that $v$ is model of a formula $F$ $(v \models F)$ if it makes $F$ true in the usual classical truth functional way. In this work, a Knowledge Base (KB) is a finite set of formulas. The language of $K$ is denoted by $\mathcal{L}(K)$ (i.e. the set of variables used in $K$ ). It is said that a $v$ is a model of $K(v \models K)$ if $v$ is a model of every formula in $K$. The set of models of $F(\operatorname{resp} . K)$ is denoted by $\operatorname{Mod}(F)(\operatorname{resp}$. $\operatorname{Mod}(K))$. Unless otherwise stated, it is assumed that $K$ is consistent.

A formula $F$ (or a $\mathrm{KB} K$ ) is consistent if $\operatorname{Mod}(F) \neq \emptyset(\operatorname{resp} \operatorname{Mod}(K) \neq \emptyset$ ). It is said that $K$ entails $F(K \models F)$ if every model of $K$ is a model of $F$, that is, $\operatorname{Mod}(K) \subseteq \operatorname{Mod}(F)$. Both notions can be naturally generalized to a KB, preserving the same notation. Two KBs $K$ and $K^{\prime}$ are equivalent $\left(K^{\prime} \equiv K\right)$, if both $K \models K^{\prime}$ and $K^{\prime} \models K$. The same notation will also be used for the equivalence with (and between) formulas.

For ease of reading, $K+F$ denotes the knowledge base that results from adding $F$.

Definition 2.1. Given two knowledge bases, $K, K^{\prime}$. It is said that:

- $K$ is an extension of $K^{\prime}$ (or $K^{\prime}$ is a retraction of $K$ ) if $\mathcal{L}\left(K^{\prime}\right) \subseteq \mathcal{L}(K)$ and

$$
\forall F \in \operatorname{Form}\left(\mathcal{L}\left(K^{\prime}\right)\right)\left[K^{\prime} \models F \quad \Longrightarrow \quad K \models F\right]
$$

- $K$ is a conservative extension of $K^{\prime}$ (or $K^{\prime}$ is a conservative retraction of $K$ ) if it is an extension that preserves the logical consequences of $K$ (in the language $\mathcal{L}\left(K^{\prime}\right)$ ),

$$
\forall F \in \operatorname{Form}\left(\mathcal{L}\left(K^{\prime}\right)\right)\left[K \models F \quad \Longrightarrow \quad K^{\prime} \models F\right]
$$

- Given $\mathcal{L}^{\prime} \subseteq \mathcal{L}(K)$, the canonical conservative retraction of $K$ to $\mathcal{L}^{\prime}$ is defined as follows:

$$
\left[K, \mathcal{L}^{\prime}\right]=\left\{F \in \operatorname{Form}\left(\mathcal{L}^{\prime}\right): K \models F\right\}
$$


Any conservative retraction on $\mathcal{L}^{\prime}$ is equivalent to the canonical one $\left[K, \mathcal{L}^{\prime}\right]$. However, since this is infinite, the problem is obtaining a finite equivalent $\mathrm{KB}$. This can be achieved by means variable forgetting.

\subsection{Conservative retractions by variable forgetting}

This section describes how to compute conservative retractions by applying variable forgetting operators. With this aim, only the notation and results that are necessary to understand the paper are presented. For a more detailed reading, as well as to consult the demonstrations, please refer to the article [43].

Definition 2.2. [43] A map

$$
\delta_{p}: \operatorname{Form}(\mathcal{L}) \times \operatorname{Form}(\mathcal{L}) \rightarrow \operatorname{Form}(\mathcal{L} \backslash\{p\})
$$

is called a variable forgetting operator for $p \in \mathcal{L}$ if

$$
\delta_{p}(F, G) \equiv[\{F, G\}, \mathcal{L} \backslash\{p\}] \quad \text { for any } F, G \in \operatorname{Form}(\mathcal{L})
$$

In other words, a variable forgetting operator for $p$ returns a formula that is equivalent to the retraction of the Knowledge Base $\{F, G\}$ to the language $\mathcal{L} \backslash\{p\}$.

A useful characterization of forgetting operators is the following: If $\delta_{p}$ is a forgetting operator for $p$, then the models of $\delta(F, G)$ are precisely the projections of models of $\{F, G\}$. Technically:

Lemma 2.3 (Lifting Lemma, [43]). Let $v: \mathcal{L} \backslash\{p\} \rightarrow\{0,1\}$ be a valuation and $\delta_{p}$ a forgetting operator for $p$. The following conditions are equivalent:

1. $v \models \delta_{p}(F, G)$

2. There exists $\hat{v}$ extension of $v$ to $\mathcal{L}$, such that $\hat{v} \models F \wedge G$

The Lifting Lemma can be also applied to $[K, \mathcal{L} \backslash\{p\}]$ [43]. Two consequences are the following useful properties:

Corollary 2.4. If $p \notin \operatorname{var}(F)$ then

$$
\delta_{p}(F, F) \equiv F \quad \text { and } \quad \delta_{p}(F, G) \equiv\left\{F, \delta_{p}(G, G)\right\}
$$

The procedure to calculate any conservative retraction is to iterate the application of the forgetting operator variables corresponding to the variables that need to be deleted from the language. Formally, it is denoted by $2^{X}$ the power set of $X$.

The natural extension of a variable forgetting operator $\delta_{p}($, ) for computing conservative retraction of KBs is one that applies $\delta_{p}(.,$.$) to all the pairs of formulas of the \mathrm{KB}$, and will be denoted by $\delta_{p}[$.$] :$

Definition 2.5. Let $\delta_{p}($, ) be a forgetting operator for $p$. It is defined

$$
\begin{aligned}
& \delta_{p}[\cdot]: 2^{\operatorname{Form}(\mathcal{L})} \rightarrow 2^{\operatorname{Form}(\mathcal{L})} \\
& \delta_{p}[K]:=\left\{\delta_{p}(F, G): F, G \in K\right\}
\end{aligned}
$$

Thus $\delta_{p}[K] \equiv[K, \mathcal{L} \backslash\{p\}]$ (see the proof in [43]). In order to keep the desired sub-language, it is sufficient to successively apply the suited operators.

When there is a variable forgetting operator for each variable, the order of variable forgetting application is not relevant from a semantic point of view since

$$
\delta_{p} \circ \delta_{q}[K] \equiv \delta_{q} \circ \delta_{p}[K]
$$

(both KBs are equivalent to $[K, \mathcal{L} \backslash\{p, q\}]$ ). When the syntactic presentation of the resulting KB (which depends on the application order) does not matter, $\delta_{Q}[K]$ express the result of applying forgetting operators for all the variables in $Q$ in some order (e.g. prefixing a linear order over language variables). sat $\delta_{\delta}(K)$ is defined as $\delta_{\mathcal{L}}[K]$, thus it has not variables and it does not depend on the operators application order. Moreover, forgetting operators are sound, if $K$ is consistent then necessarily $\operatorname{sat}_{\delta}(K)=\{\top\}$. In fact, $K$ is inconsistent if and only if $\perp \in \operatorname{sat}_{\delta}(K)$ (see [43]).

Since

$$
\delta_{\mathcal{L} \backslash \operatorname{var}(F)}[K] \equiv[K, \operatorname{var}(F)]
$$

the following useful property is straightforward: 
Corollary 2.6 (Location Property, [44]).

$$
K \models F \quad \Longleftrightarrow \quad \delta_{\mathcal{L} \backslash \operatorname{var}(F)}[K] \models F
$$

An approach to specify contexts in $\mathrm{AI}$ is based on determining which set of variables c $\subseteq \mathcal{L}$ provides non-trivial information and which variables are relevant for representing the specific context. Note that in context-based reasoning formalizations, the contexts are often determined by a variable set (for example, the variables which represent signals received by the agent). In this way, a context is identified with a sublanguage of $\mathcal{L}$. Then the state of the context is determined by the truth values of the variables of such sublanguage. For example

$$
\mathbf{c}=\left\{4 G \_ \text {mode, home, bluetooth_colleague }\right\}
$$

could represent the context where mobile devices work only with signals from a telecom operator, there is a sensor that detects whether the user is at home (for example, by recognizing home WiFi network) and there is a Bluetooth signal from a friend. A state of the context is the truth values from variables of $\mathbf{c}$.

When $K$ does not provide any specific knowledge about the context specified by $Q$, it is natural to conclude that $[K, Q]$ should contain only tautologies (in the language $Q$ ), hence $\delta_{\mathcal{L} \backslash Q}[K] \equiv\{\top\}$.

Definition 2.7. Let $\mathcal{L}$ be a propositional language and $\mathcal{L}_{0} \subseteq \mathcal{L}$ a sublanguage.

- $K_{\mid \mathcal{L}_{0}}$ denotes a conservative retraction of $K$ to $\mathcal{L}_{0}$, that is, a KB such that

$$
K_{\uparrow \mathcal{L}_{0}} \equiv\left[K, \mathcal{L}_{0}\right]
$$

or, by means of forgetting operators, $K_{\left\lceil\mathcal{L}_{0}\right.} \equiv \delta_{\mathcal{L} \backslash \mathcal{L}_{0}}[K]$

- Given $F \in F \operatorname{orm}(\mathcal{L}), F_{\uparrow \mathcal{L}_{0}}$ is defined as a conservative retraction of $\{F\}$ to $\mathcal{L}_{0}$.

From a semantic point of view (and also bearing in mind that our main purpose is to establish both the formal foundations of the problem and solutions), the syntactic representation chosen to work with the above definitions is not relevant, since they are all equivalent. Therefore, it would suffice to select forgetting operators to obtain $F_{\mid \mathcal{L}_{0}}$. In the next section an example of this type of operators is presented.

A useful property is the following:

Proposition 2.8. $K \models F \quad \Longrightarrow \quad K_{\uparrow \mathcal{L}_{0}} \models F_{\uparrow \mathcal{L}_{0}}$

Proof. By reductio ad absurdum, let us suppose that there exists

$$
v: \operatorname{Form}\left(\mathcal{L}_{0}\right) \rightarrow\{0,1\}
$$

such that $v \models K_{\uparrow \mathcal{L}_{0}}+\neg F_{\uparrow \mathcal{L}_{0}}$. This means that $v$ can be extended to a model of $K$ but not to a model of $F$. However, if $\hat{v}$ is an

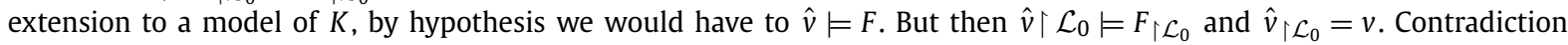

\subsection{Canonical forgetting operator}

Recall that $\delta_{p}(K) \equiv[K, \mathcal{L} \backslash\{p\}]$ and therefore forgetting operators for the same variable produces equivalent KBs. In order to illustrate some of the results with examples, this section presents a particular type of forgetting operators. In [43], authors show a class of forgetting operators following this idea, called canonical forgetting operators. Although they could not be efficient for computing conservative retractions in complex cases, from a theoretical point of view, they are equally useful and easier to describe than those of algebraic nature introduced in the same paper.

Structurally, the operator in turn consists of two maps. The first one forgets the variable using the Lifting Lemma and the second one removes the occurrences of the constants $\perp$ and $T$ in the resulting formula. The second one is a reducer operator,

$$
\sigma: \operatorname{Form}(\mathcal{L}) \rightarrow \operatorname{Form}^{r}(\mathcal{L})
$$

$\left(F \operatorname{orm}^{r}(\mathcal{L})\right.$ is the set of propositional formulas with no occurrences of $\left.\perp, \top\right)$ such that $\sigma(F) \equiv F$ for any $F$. The canonical forgetting operator for the variable $p$ is $\delta_{p}^{0}=\sigma \circ \delta_{p}^{*}$ where

$$
\delta_{p}^{*}(F, G):=(F \wedge G)\{p / \top\} \vee(F \wedge G)\{p / \perp\}
$$




\section{Knowledge Harnessing}

In this section the concept of knowledge harnessing is formalized. A fundamental requirement is that, when the information is restricted to what can be expressed in a language, then the obtained information is non-trivial.

\section{Definition 3.1.}

- A language $\mathcal{L}_{0}$ is informative for $F$ if $F_{\mid \mathcal{L}_{0}} \not \equiv T$.

- Let $K$ be a $\mathrm{KB}, F$ be a formula and $\mathcal{L}_{0} \subseteq \mathcal{L}$ a sublanguage. It is said that $F$ contains harnessed $\mathcal{L}_{0}$-knowledge with respect to $K$, (notation: $K \models_{\mathcal{L}_{0}} F$ ) if $\mathcal{L}_{0}$ is informative for $F$ and $K \models F_{\uparrow \mathcal{L}_{0}}$.

Remark 3.2. Let us consider deliberative agents. If the agent works with knowledge $K$ (in the sense of GOLOG, for example), then its KB changes only if he/she infers such change from the knowledge he has about actions and their effects (reasoning by progression), or because it receives external information that should be accepted, i.e. that at least does not cause inconsistency with his/her knowledge.

If the agent receives $F$ that contains harnessed $\mathcal{L}_{0}$-knowledge with respect to its $\mathrm{KB}$, then $K \models F_{\uparrow \mathcal{L}_{0}}$ so the assimilation of $F_{\uparrow \mathcal{L}_{0}}$ does not cause inconsistency and would allow the validation of preconditions for new actions.

The idea behind the definition is that, in the usual case where $\mathcal{L}_{0}$ specifies a concept, the formula $F_{\left\lceil\mathcal{L}_{0}\right.}$ takes up the context information that is valid according to $K$. Following with this idea, an interesting case for Knowledge Harnessing occurs when $K+F$ is inconsistent but $K=_{\mathcal{L}_{0}} F$. This happens, for instance, when an agent receives (from other agent) information that is inconsistent with its beliefs, represented by $K$, but it can validate the information about the context specified in the language $\mathcal{L}_{0}$

If, in addition, $K+F$ was inconsistent we have that $K \models \neg F$, thus $K \models(\neg F)_{\mathcal{L}_{0}}$ by Location Lemma. Therefore the situation could be described by the logical condition

$$
K \models F_{\left\lceil\mathcal{L}_{0}\right.} \wedge(\neg F)_{\uparrow \mathcal{L}_{0}}
$$

the agent selects $F_{\mid \mathcal{L}_{0}}$ and discards $(\neg F)_{\mid \mathcal{L}_{0}}$ because it is assumed that the agent has confidence in the values of the sensors represented by $F$. That is why $F_{\mid \mathcal{L}_{0}}$ is chosen as knowledge (belief) about the context to use in the decision making process. From the logical point of view, and without sticking to that trust, the knowledge base $K$ can not decide between $F_{\uparrow \mathcal{L}_{0}}$ and $(\neg F) \mathcal{L}_{0}$.

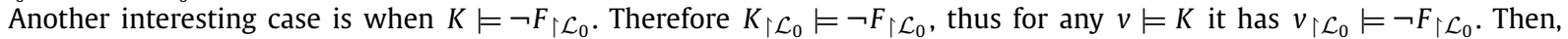
by Lifting Lemma there is not any extension of $v_{\mid \mathcal{L}_{0}}$ to model $F$. In particular $v$ itself is an extension of $v_{\uparrow \mathcal{L}_{0}}$ so $v \models \neg F$. Therefore $K \models \neg F$.

The second condition in the Proposition bellow characterizes knowledge harnessing in terms of conservative retraction (hence, in variable forgetting terms).

Proposition 3.3. Let $F \in \operatorname{Form}(\mathcal{L}(K))$. The following properties hold for harnessed knowledge:

1. If $K \models F$ then $K \models \mathcal{L}_{0} F$ for any sublanguage $\mathcal{L}_{0}$ of $\mathcal{L}(K)$

2. $K \models \mathcal{L}_{0} F$ if and only if $\left[K, \mathcal{L}_{0}\right] \models F_{\curlyvee \mathcal{L}_{0}}$

Proof. (1) is true because $F \models F_{\mid \mathcal{L}_{0}}$

To prove (2), it is sufficient to see that the variables that occur in $F_{\mid \mathcal{L}_{0}}$ belong to $\mathcal{L}_{0}$, thus by Location Lemma $K \models F_{\mid \mathcal{L}_{0}}$ holds if and only if $\left[K, \mathcal{L}_{0}\right]=F_{\mid \mathcal{L}_{0}}$.

Note that the reciprocal of $(1)$ is trivial since $\mathcal{L}(K)$ itself is one of such sublanguages and the retraction of $F$ to this is $F$ itself. However, if we restrict ourselves to the proper sublanguages, the reciprocal is not true: Consider

$$
K=\{\neg p \wedge \neg q\} \text {, and } F=p \vee q
$$

The proper sublanguages are $\mathcal{L}_{1}=\{p\} \mathcal{L}_{2}=\{q\}$. For both languages $F_{\mid L_{i}} \equiv T$, thus $K$ entails them, but $K \forall p \vee q$.

Example 3.4. In this example the computations of conservative retractions have been omitted in order to make it easier to read (see [43]).

Let $G=s \rightarrow r$ and $K$ be the KB

$$
K=\left\{\begin{array}{l}
t \wedge p \leftrightarrow s \\
t \wedge r \rightarrow s \\
t \wedge q \rightarrow s \\
p \wedge q \wedge s \wedge t \rightarrow r
\end{array}\right.
$$


In this case $G_{\{\{r\}} \equiv G_{\{\{s\}} \equiv T$ thus both are not informative, although $\{r, s\}$ does since $G_{\{\{r, s\}}=G$. However

$$
[K,\{r, s\}] \equiv\{\top\} \not \models G
$$

because, by applying location lemma,

$$
\delta_{\mathcal{L} \backslash\{r, s\}}[K] \equiv \delta_{p}\left[\delta_{q}\left[\delta_{t}[K]\right]\right]=\{\top\}
$$

It is, therefore, an example of the worst case for context reasoning: it is not possible to extract useful information from $G$, and in this case the reason is that $K$ itself does not provide useful information about the two possible contexts in the language $\{r, s\}$.

Let us consider now the formula

$$
F=p \wedge q \wedge t \rightarrow s
$$

In this case $K \models \mathcal{L}_{0} F$ because $K \models F$ and $F_{\mid \mathcal{L}_{0}} \not \equiv \top$ for any $\mathcal{L}_{0} \subseteq\{p, q, t, s\}$.

Let us consider now

$$
H=t \wedge p \leftrightarrow r
$$

and $\mathcal{L}_{0}=\{t, r\}$. In this case $K \not \models H$, although $K \models\{t, r\}$ since $H_{\uparrow\{t, r\}} \equiv t \leftrightarrow r$ and $K_{\left\lceil\mathcal{L}_{0}\right.}$ entails this formula.

\subsection{Informative languages}

Before defining the TWD model, some properties of the informative languages will be examined.

Definition 3.5. The set of informative sublanguages for $F$ with respect to $\mathcal{L}$ is

$$
\mathbb{I}_{\mathcal{L}}(F)=\left\{\mathcal{L}_{0} \subseteq \mathcal{L}: F_{\left\lceil\mathcal{L}_{0}\right.} \not \equiv \top\right\}
$$

The following proposition summarizes the main properties of the set of informative languages for a given formula.

Proposition 3.6. The following properties hold:

1. If $\operatorname{var}(F) \subseteq \mathcal{L}_{0}$ and $F$ is not a tautology, then $\mathcal{L}_{0} \in \mathbb{I}_{\mathcal{L}}(F)$

2. $\mathcal{L}_{0} \subseteq \mathcal{L}_{1}$ and $\mathcal{L}_{0} \in \mathbb{I}_{\mathcal{L}}(F)$ then $\mathcal{L}_{1} \in \mathbb{I}_{\mathcal{L}}(F)$

3. $\mathbb{I}_{\mathcal{L}}(F)$ is closed under union

Proof. (1) is true since, in this case, $F_{\mid \mathcal{L}_{0}}=F$

Proof of (2): If $\mathcal{L}_{0} \in \mathbb{I}_{\mathcal{L}}(F)$ then $F_{\mid \mathcal{L}_{0}} \not \equiv \top$. Since, in this case, $\mathcal{L}_{0} \subseteq \mathcal{L}_{1}$, trivially $F_{\mid \mathcal{L}_{0}} \equiv\left(F_{\uparrow \mathcal{L}_{1}}\right)_{\mid \mathcal{L}_{0}}$, that is, $F_{\mid \mathcal{L}_{0}}$ is a con-

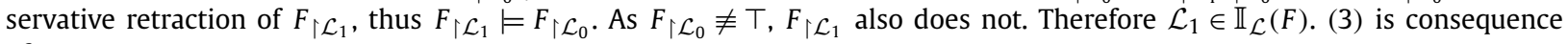
of (2).

\section{A model of three-way decision for Knowledge Harnessing}

In order to estimate how reliable is, from a semantic point of view, the information that $F$ contains about a given context, an evaluation approach is proposed. In this approach reliability is understood in a similar way to confidence in association rules: the number of models of the KB that validate this information (Fig. 2). In this way, the engineer is provided with an scale and acceptance thresholds for the border region can be established on it.

Definition 4.1. Let $K$ be a (consistent) $\mathrm{KB}$ and $\mathcal{L}_{0} \subseteq \mathcal{L}(K)$. The acceptable-rejection evaluation for harnessing w.r.t. $K$ and $\mathcal{L}_{0}$ is the function

$$
\omega_{\mathcal{L}_{0}}^{K}: \operatorname{Form}(\mathcal{L}) \rightarrow[0,1]
$$

defined by

$$
\omega_{\mathcal{L}_{0}}^{K}(F)=\frac{\mid\left\{v \in \operatorname{Mod}(K): v\left\lceil_{\mathcal{L}_{0}} \models F_{\left\lceil\mathcal{L}_{0}\right.}\right\} \mid\right.}{|\operatorname{Mod}(K)|}
$$

Proposition 4.2. The following conditions hold

1. $\omega_{\mathcal{L}_{0}}^{K}(F)=1 \Longleftrightarrow K==_{\mathcal{L}_{0}} F$ 


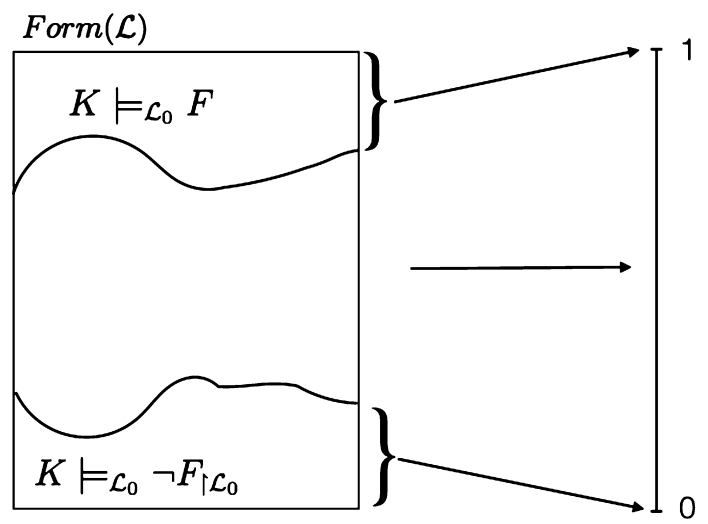

Fig. 2. Idea of acceptance-rejection evaluations on formulas.

2. $\omega_{\mathcal{L}_{0}}^{K}(F)=0 \Longleftrightarrow K \models \mathcal{L}_{0} \neg F_{\mid \mathcal{L}_{0}}$

3. $0<\omega_{\mathcal{L}_{0}}^{K}(F)<1 \Longleftrightarrow K \not \forall F_{\mid \mathcal{L}_{0}}$ and $K \not \forall \neg F_{\mid \mathcal{L}_{0}}$

Proof. (1):

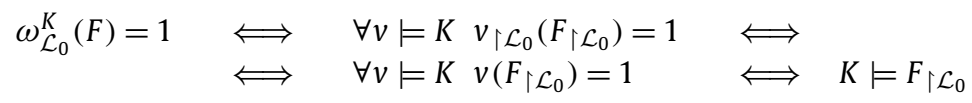

The proof of (2) is similar:

$$
\begin{aligned}
& \omega_{\mathcal{L}_{0}}^{K}(F)=0 \quad \Longleftrightarrow \quad \forall v \models K v_{\uparrow \mathcal{L}_{0}}\left(F_{\left\lceil\mathcal{L}_{0}\right.}\right)=0 \quad \Longleftrightarrow
\end{aligned}
$$

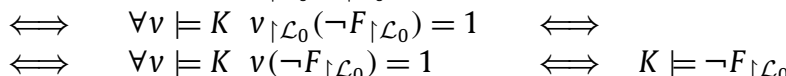

(3) is consequence of (1), (2).

Once the acceptance-rejection evaluation is defined, the sets $P O S_{\mathcal{L}_{0}}^{K}, N E G_{\mathcal{L}_{0}}^{K}$ and $B N D_{\mathcal{L}_{0}}^{K}$ are defined in the usual way [35], that is:

- $P O S_{\mathcal{L}_{0}}^{K}=\left(\omega_{\mathcal{L}_{0}}^{K}\right)^{-1}(1)$

- $N E G_{\mathcal{L}_{0}}^{K}=\left(\omega_{\mathcal{L}_{0}}^{K}\right)^{-1}(0)$

- $B N D_{\mathcal{L}_{0}}^{K}=\left(\omega_{\mathcal{L}_{0}}^{K}\right)^{-1}((0,1))$

The acceptance-rejection evaluation for harnessing w.r.t. $K$ and $\mathcal{L}_{0}$ combines two important dimensions. On the one hand, it takes into account the representation language of that information $\left(\mathcal{L}_{0}\right)$ and on the other hand, the number of $K$ models where the harnessed information is valid with respect to that language. A high value is estimating how prudent is, for an agent, to accept that harnessed information (since it is valid in a higher number of possible states of the agent). If that value is 0 then an inconsistency has been created. Seen from the non-monotonous reasoning point of view, $\omega_{\mathcal{L}_{0}}^{K}(F)$ is a measure of how many models are preserved, given a sub-language. The language choice will be later discussed.

For instance, such an evaluation for agents based on the Situation Calculus it is possible to keep the value of $\omega_{\mathcal{L}_{0}}^{K}(F)$ associated to the harnessed knowledge $F_{\mid \mathcal{L}_{0}}$ of a fluent that comes from outside (coming from sensors or monitoring of external events). So, for example, if the agent uses $F_{\left\lceil\mathcal{L}_{0}\right.}$ to project the initial situation through a high-level action program, a value of robustness using the values can be associated to the fluents of the projected situation (the database that results after performing such program). It is clear that, in that case, it will be very likely to combine both, different harnessed fluents and sub-languages, so it is necessary to combine them. In the case of teleo-reactive agents it would allow to simplify the TR-successions, in exchange for paying the price of taking into account that the action dictating the succession has a degree of acceptance.

\subsection{Behavior of three-way decisions by extending the knowledge base}

The following proposition summarizes the basic cases and properties of the decision regions when $\mathcal{L}_{0}=\mathcal{L}$, as well as the behavior of the evaluation by expansion of $K$ with new information under the sublanguage. This case is interesting as it represents the situation in which one receives new knowledge about the context of the system KB. 
Proposition 4.3. The following properties hold for $\omega_{\mathcal{L}}^{K}$

1. If $K$ is an extension of $K^{\prime}$ then
(a) $\operatorname{POS}_{\mathcal{L}}^{K^{\prime}} \subseteq \operatorname{POS}_{\mathcal{L}}^{K}$
(b) $N E G_{\mathcal{L}}^{K^{\prime}} \subseteq N E G_{\mathcal{L}}^{K}$
(c) $B N D_{\mathcal{L}}^{K} \subseteq B N D_{\mathcal{L}}^{K^{\prime}}$

2. If $F \in \operatorname{Form}\left(\mathcal{L}_{0}\right)$ then $\omega_{\mathcal{L}_{0}}^{K+F}(G) \geq \omega_{\mathcal{L}_{0}}^{K}(F \wedge G)$

Proof. Property (1).1 is true since, by Proposition 4.2.(1), if $F \in P O S_{\mathcal{L}}^{K^{\prime}}$ then $K^{\prime} \models F_{\mid \mathcal{L}_{0}}$ hence $K \models F_{\left\lceil\mathcal{L}_{0}\right.}$ therefore (by Proposition 4.2.(1) again) $\omega_{\mathcal{L}_{0}}^{K}(F)=1$.

Both (1).2 and (1).3 are proved as (1).1.

Proof of (2) Please note that in this case, by Corollary 2.4, it holds that

$$
\begin{aligned}
& (F \wedge G)_{\left\lceil\mathcal{L}_{0}\right.} \equiv F \wedge G_{\left\lceil\mathcal{L}_{0}\right.} \equiv\left(F \wedge G_{\uparrow \mathcal{L}_{0}}\right)_{\uparrow \mathcal{L}_{0}} \\
& \omega_{\mathcal{L}_{0}}^{K+F}(G)=\frac{\mid\left\{v \models K+F: v_{\left\lceil L_{0}\right.} \models G_{\left\lceil\mathcal{L}_{0}\right\}}\right\}}{|\operatorname{Mod}(K+F)|} \geq \\
& \geq \quad \frac{\left|\left\{v \models K+F: v_{\left\lceil L_{0}\right.} \models G_{\mid \mathcal{L}_{0}}\right\}\right|}{|\operatorname{Mod}(K)|}= \\
& =\quad \frac{\left|\left\{v \models K: v \models F \wedge G_{\left\{\mathcal{L}_{0}\right.}\right\}\right|}{|\operatorname{Mod}(K)|}= \\
& \text { (by Cor. 2.4) } \frac{\mid\left\{v \models K: v_{\mid L_{0}}=\left(F \wedge G_{\mid \mathcal{L}_{0}}\right)_{\left.\mid \mathcal{L}_{0}\right\} \mid}\right.}{|\operatorname{Mod}(K)|}= \\
& =\quad \omega_{\mathcal{L}_{0}}^{K}(F \wedge G)
\end{aligned}
$$

\subsection{Behavior of three-way decisions by extending the language}

In this section the behavior of the acceptance-rejection evaluations when there exists an extension of the language is studied. It would correspond to the case in which the context (the set of variables) is extended or combined with another one. This case would correspond to the situation in which the agent must combine different harnessed information (which he/she possesses with respect to different situations) in order to be able to decide the action to be executed and to estimate the degree of acceptance-rejection of the result. In order to establish the relationship between the original evaluations and those of the extension, two operations on the evaluations have been defined (which are simply a rewriting of the minimum norm and maximum conorm of Fuzzy Logic).

\section{Definition 4.4.}

- $\omega_{\mathcal{L}_{1}}^{K} \vee \omega_{\mathcal{L}_{2}}^{K}=\max \left\{\omega_{\mathcal{L}_{1}}^{K}, \omega_{\mathcal{L}_{2}}^{K}\right\}$

- $\omega_{\mathcal{L}_{1}}^{K} \wedge \omega_{\mathcal{L}_{2}}^{K}=\min \left\{\omega_{\mathcal{L}_{1}}^{K}, \omega_{\mathcal{L}_{2}}^{K}\right\}$

Theorem 4.5. Let $\mathcal{L}_{1}, \mathcal{L}_{2}$ be sublanguages of $\mathcal{L}=\mathcal{L}(K)$.

1. $\mathcal{L}_{1} \subseteq \mathcal{L}_{2} \Longrightarrow \omega_{\mathcal{L}_{2}}^{K} \leq \omega_{\mathcal{L}_{1}}^{K}$

2. $\omega_{\mathcal{L}_{1} \cup \mathcal{L}_{2}}^{K} \leq \omega_{\mathcal{L}_{1}}^{K} \wedge \omega_{\mathcal{L}_{2}}^{\mathcal{K}^{2}}$

3. $\omega_{\mathcal{L}_{1} \cap \mathcal{L}_{2}}^{K} \geq \omega_{\mathcal{L}_{1}}^{K} \vee \omega_{\mathcal{L}_{2}}^{K}$

Proof. Let us see (1):

$$
\begin{aligned}
\omega_{\mathcal{L}_{2}}^{K}(F)= & \frac{\mid\left\{v \in \operatorname{Mod}(K): v_{\left\lceil\mathcal{L}_{2}\right.} \models F_{\left\lceil\mathcal{L}_{2}\right\} \mid}\right.}{|\operatorname{Mod}(K)|} \leq \\
& \leq \frac{\mid\left\{v \in \operatorname{Mod}(K): v_{\left\lceil\mathcal{L}_{2}\right.} \models F_{\left\lceil\mathcal{L}_{1}\right\} \mid}\right.}{|\operatorname{Mod}(K)|} \quad \text { (because } F_{\left\lceil\mathcal{L}_{2}\right.} \models F_{\left\lceil\mathcal{L}_{1}\right.} \text { ) }
\end{aligned}
$$


(2) and (3) holds since by property (1)

$$
\omega_{\mathcal{L}_{1} \cup \mathcal{L}_{2}}^{K}(F) \leq \omega_{\mathcal{L}_{i}}^{K}(F) \text { and } \omega_{\mathcal{L}_{1} \cap \mathcal{L}_{2}}^{K}(F) \geq \omega_{\mathcal{L}_{i}}^{K}
$$

Example 4.6. The inequalities of the above proposition can be strict. For instance, let us see it for (2): Let

$$
K=\{\neg p \vee \neg q\}, \mathcal{L}_{1}=\{p\} \text { and } \mathcal{L}_{2}=\{q\}
$$

In this case $|\operatorname{Mod}(K)|=3$.

Let us consider $F=p \wedge q$. Then $F_{\uparrow \mathcal{L}_{1}} \equiv p$ and $F_{\uparrow \mathcal{L}_{2}}=\equiv q$. Therefore

$$
\omega_{\{p\}}^{K}(F)=\omega_{\{q\}}^{K}(F)=1 / 3
$$

However $F_{\left\lceil\mathcal{L}_{1} \cup \mathcal{L}_{2}\right.} \equiv F$ and $\omega_{\{p, q\}}^{K}(F)=0$

The problem of calculating the evaluation is a computationally complex problem. Therefore, it would be interesting to do the calculations with languages (contexts) for which the associated evaluation had a maximum value for the $F$ formula among all contexts. The idea is to try to estimate what is the maximum amount of information (depending on the size of the sublanguage, which would correspond to the corresponding number of sensors in the previous examples) that can be harnessed (being of course, an informative language). Formally:

\section{Definition 4.7.}

1. $\Omega^{K}(F)=\left\{\begin{array}{lr}\max \left\{\omega_{\mathcal{L}_{0}}^{K}(F): \mathcal{L}_{0} \text { is informative for } F\right\} & \text { if it is nonempty } \\ 0 & \text { in other case }\end{array}\right.$

2. It is said that $\mathcal{L}_{0}$ is maximally informative for $F$ with respect to $K$ if $\Omega^{K}(F)=\omega_{\mathcal{L}_{0}}^{K}(F)$ with the size of $\mathcal{L}_{0}$ being maximum

Following with the Example 4.6, it holds that $\{p\}$ and $\{q\}$ are maximally informative but $\{p, q\}$ does not.

When $\Omega^{K}(F)=0$ there is not informative language $\mathcal{L}_{0}$ verifying that $K \models \mathcal{L}_{0} F$ therefore it can not extract from $F$ any information. In case of $\Omega^{K}(F)=1$ then there exists harnessed knowledge for some sublanguage. Finally, if $0<\Omega^{K}(F)<1$ the function estimates the maximal information potentially usable in some models of $K$.

The following theorem shows the importance of maximally informative languages: their sub-languages (subcontexts, in the case of context-based reasoning) do not provide more information regarding the associated evaluations:

Theorem 4.8. Let $\mathcal{L}_{1} \subseteq \mathcal{L}_{0}$ be two informative languages for $F$, such that $\Omega^{K}(F)=\omega_{\mathcal{L}_{0}}^{K}(F)$. Then

1. $\omega_{\mathcal{L}_{0}}^{K}(F)=\omega_{\mathcal{L}_{1}}^{K}(F)$

2. $K \stackrel{\mathcal{L}_{0}}{=} F_{\uparrow \mathcal{L}_{0}} \leftrightarrow F_{\uparrow \mathcal{L}_{1}}$

Proof. (1) is true since $\omega_{\mathcal{L}_{0}}^{K}(F) \leq \omega_{\mathcal{L}_{1}}^{K}(F)$ by Theorem 4.5. As $\omega_{\mathcal{L}_{0}}^{K}(F)$ is maximum, the equality holds.

Proof of (2). By definition of $\omega_{\mathcal{L}}^{K}$, and as $\mathcal{L}_{1} \subseteq \mathcal{L}_{0}$ :

$$
\left\{v \models K: v_{\mid \mathcal{L}_{0}}=F_{\left\lceil\mathcal{L}_{0}\right.}\right\} \subseteq\left\{v \models K: v_{\left\lceil\mathcal{L}_{1}\right.} \models F_{\left\lceil\mathcal{L}_{1}\right.}\right\}
$$

Since the first set has maximum size, the equality holds, thus $K \models F_{\uparrow \mathcal{L}_{0}} \leftrightarrow F_{\uparrow \mathcal{L}_{1}}$.

As a consequence of the above result, maximally informative language can not be combined. The following corollary shows that it is possible to choose a maximally informative language within a given context, but it will not be possible to combine it with others (that are associated to other contexts) since it would not be harnessed knowledge.

Corollary 4.9. If $\mathcal{L}_{1} \neq \mathcal{L}_{0}$ are maximally informative for $F$ then $\mathcal{L}_{1} \cup \mathcal{L}_{0}$ does not.

Proof. Since both languages are of maximum size, $\mathcal{L}_{1} \not \subset \mathcal{L}_{0}$ and $\mathcal{L}_{0} \not \subset \mathcal{L}_{1}$ holds. Therefore $\left|\mathcal{L}_{1} \cup \mathcal{L}_{0}\right|>\left|\mathcal{L}_{0}\right|$. By Theorem 4.5

$$
\omega_{\mathcal{L}_{1} \cup \mathcal{L}_{0}}^{K}(F) \leq \omega_{\mathcal{L}_{0}}^{K}(F)
$$

However, the inequality has to be strict as $\left|\mathcal{L}_{0}\right|$ is maximum. 

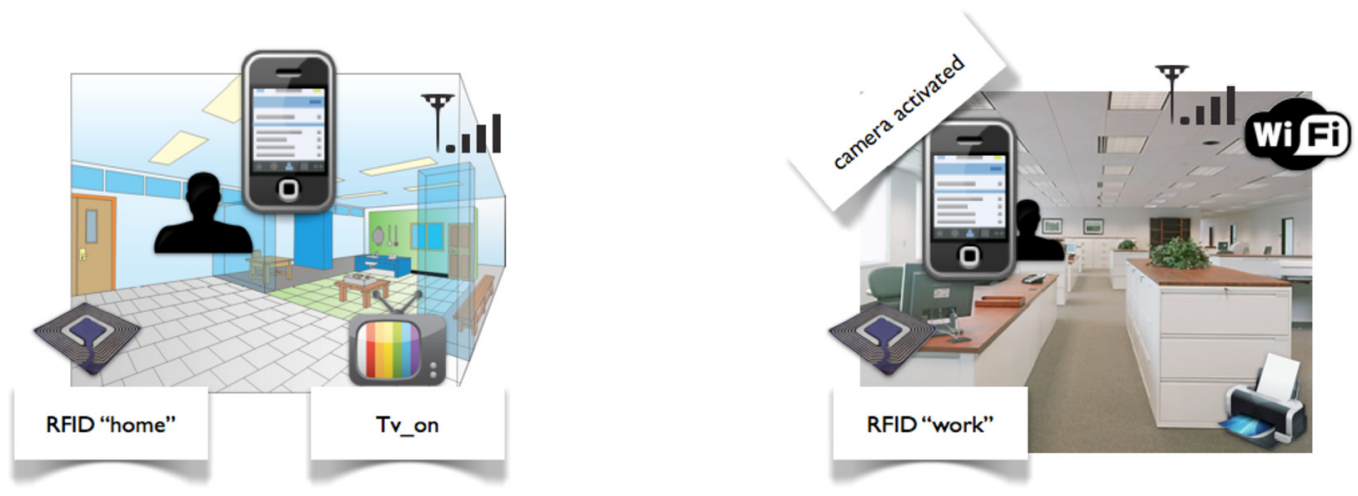

Fig. 3. Two contexts for the Knowledge Base.

Following with Example 4.6,

$$
\Omega_{\{p\}}^{K}(F)=\Omega_{\{q\}}^{K}(F)=1 / 3
$$

Since $\{p, q\}$ is not informative, both languages are maximal.

\section{Examples}

In order to illustrate the formalization, two more developed examples are presented below. The first one is also within the framework of mobile MAS from Sect. 1.1. The second one rewrites an example of [45].

\subsection{An use case in MAS-based mobile context reasoning}

This section details a particular example within the framework described in Sect. 1.2. The framework described below is motivated by the design of semantic apps in the context of the mobile Web 2.0 [10]. The scenario involves a context-aware mobile phone that has been configured to decide mobile phone's behavior, based on user's preferences and context. One of the services the system provides, MMS service, is paid per use, so the KB is designed to restrict its use to contexts where there are not alternative ways to send multimedia content (where there is not a supplementary cost per use as for example WiFi).

For instance, let $K_{f}$ be a knowledge base built to work only within two contexts representing home and work environments (see Fig. 3). In the general case, a global KB must represent features about every signal relevant for the user. For a sound behavior at home, the agent should get a contextualized KB from $K_{f}$ of the server to work properly in each context (by conservative retraction). For example, contexts depicted in Fig. 3 can be described by means of the variable sets (set of propositional variables related to signals in each context):

- $\mathcal{L}_{0}=\left\{\right.$ home, $\left.t v \_o n, 4 G\right\}$, and

- $\mathcal{L}_{1}=\{$ work, bluetooth_printer, $4 G$, WiFi $\}$.

The state of the context is specified by giving the truth value of the associated variables. Let $K$ be one of the corresponding conservative retractions. If the user of other device provides information $F$ to the mobile phone, it could be interesting to see if $K_{\uparrow \mathcal{L}_{0}} \models \mathcal{L}_{0} F_{\left\lceil\mathcal{L}_{0}\right.}$, (or for $\mathcal{L}_{1}$ ). In the case of mobile reasoning, the conservative retraction has to preserve information about mobile features. In this case, according to $K_{f}$, it is represented by a language $\mathcal{L}_{a}$,

$$
\begin{aligned}
\mathcal{L}_{a}= & \{\text { block_work_calls, ringtone_1,ringtone_2, mute_mode, VoIP_on, } \\
& \text { 4G_mode,tag_home_set,tag_work_set, printer_flag, } \\
& \text { mowento_mms, connectivity }\}
\end{aligned}
$$

In order to illustrate the use of conservative retraction for knowledge harnessing, let us suppose that the user is at home, the TV is off, and a colleague arrives with the Bluetooth signal turned on. Thus, the context to study is

$$
\mathcal{L}_{0}=\left\{4 G \_ \text {mode, } t v \_o n, \text { home, bluetooth_colleague }\right\}
$$

And therefore, the conservative retraction to reason within this context that has to be computed is

$$
K_{h}=\left[K, \mathcal{L}_{a} \cup\left\{\text { home }, t v \_o n, \text { bluetooth_colleague, } 4 G \_ \text {mode }\right\}\right]
$$


- If user is at home, agent blocks incoming working calls:

$F_{1}:$ home $\rightarrow$ block_work_calls

- If user is at home, then ringtone ${ }_{1}$ is activated; If user is at work, then ringtone 2 is activated:

$F_{3}:$ home $\rightarrow$ ringtone $_{1}, F_{2}:$ work $\rightarrow$ ringtone $_{2}$

- User does not work at home:

$F_{4}:$ work $\rightarrow \neg$ home

- Both ringtones can not be activated at the same time:

$F_{5}:$ ringtone $_{1} \rightarrow \neg$ ringtone $_{2}$

- When user is at home and TV is on, mobile mute mode must be on:

$F_{6}:$ home $\wedge t v \_o n \rightarrow$ mute_mode

- If TV is off, then mute mode is off, too:

$F_{7}: \neg t v_{-}$on $\rightarrow \neg$ mute_mode

- When agent is not connected to WiFi network, and user is at work, VoIP service is launched and 4G mode is turned off:

$F_{8}:$ work $\wedge$ wifi $\rightarrow$ VoIP_on $\wedge \neg 4 G \_$mode

- If a colleague's Bluetooth device is detected, then agent believes that user is at work:

$F_{9}:$ bluetooth_colleague $\rightarrow$ work

- If user takes a picture at home (at work), then the home (work) tagging is activated.

$F_{10}:$ photo $\wedge$ home $\rightarrow$ tag_home_set, $F_{11}:$ photo $\wedge$ work $\rightarrow$ tag_work_set

- If agent detects printer's Bluetooth signal, then printer_flag is on:

$F_{12}:$ bluetooth_printer $\rightarrow$ printer_flag

- If agent can not connect to WiFi access point, then an specific service called Mowento's MMS service is launched:

$F_{13}: \neg$ WiFi $\wedge$ photo $\rightarrow$ mowento_mms

- If neither WiFi or $4 \mathrm{G}$ are available, then no connection warning is activated:

$F_{14}: \neg$ wifi $\wedge \neg 4 G \_$mode $\rightarrow \neg$ connectivity

- When user is at work, only one (4G or VoIP) could be activated:

$F_{15}:$ work $\rightarrow \neg\left(4 G \_\right.$mode $\wedge$ VoIP $)$

Fig. 4. Global KB for the example of Fig. 3.

- bluetooth_colleague $\rightarrow$ ringtone $_{2}$

- bluetooth_colleague $\wedge$ home $\rightarrow \perp$

- bluetooth_colleague $\wedge 4 G \_$mode $\rightarrow \neg V o I P$

- bluetooth_colleague $\rightarrow$ tag_work_set $\vee \neg$ photo

- bluetooth_colleague $\wedge \neg 4 G \_$mode $\rightarrow V$ oIP $\vee \neg$ connectivity

- 4G_mode $\wedge$ blue $\rightarrow \neg V o I P \vee \neg$ photo

- 4G_mode $\wedge$ blue $\rightarrow$ VoIP $\vee \neg$ photo

- bluetooth_colleague $\wedge \neg 4 G \_$mode $\rightarrow \neg$ photo $\vee V o I P$

Fig. 5. Formulas of the contextualization of the KB from Fig. 4 to home context.

(Note that $\mathcal{L}_{s}=\mathcal{L}_{a} \cup\left\{\right.$ home, $t v \_o n$, bluetooth_colleague, $4 G \_$mode $\}$.) That is,

$\delta_{\{\text {work,WiFi,bluetooth_printer }\}}[K]$

The conservative retraction $K_{h}$ contains the set

$$
\left\{F_{1}, F_{3}, F_{5}, F_{6}, F_{7}, F_{10}\right\}
$$

from the initial $\mathrm{KB}$, jointly with the formulas depicted in Fig. 5.

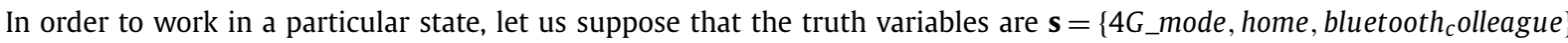
and that the mobile agent receives the information

$$
F \equiv \text { bluetooth_colleage } \wedge \text { home } \wedge \text { VoIP } \wedge \neg \text { work }
$$

To harness knowledge from $F$, the formula $F_{\left\lceil\mathcal{L}_{s}\right.}$ needs to be computed:

$$
F_{\uparrow \mathcal{L}_{s}} \equiv \text { bluetooth_colleage } \wedge \text { home } \wedge \neg \text { work }
$$

In this case $K_{h}+\mathbf{s} \models F_{\left\lceil\mathcal{L}_{s}\right.}$, that is,

$$
K \models \mathcal{L}_{S} F
$$

(and thus $\omega_{\mathcal{L}_{a}}^{K}(F)=1$ ). 


$$
\begin{array}{ll}
\text { ok_pump } \wedge \text { on_pump } \Rightarrow \text { water } & \text { man_fill } \Rightarrow \text { water } \\
\text { man_fill } \Rightarrow \neg \text { on_pump } & \neg \text { man_fill } \Rightarrow \text { on_pump } \\
\text { water } \wedge \text { ok_boiler } \wedge \text { on_boiler } \Rightarrow \text { steam } & \neg \text { water } \Rightarrow \neg \text { steam } \\
\neg \text { ok_boiler } \Rightarrow \neg \text { steam } & \neg \text { on_boiler } \Rightarrow \neg \text { steam } \\
\text { steam } \wedge \text { coffee } \Rightarrow \text { hot_drink } & \text { coffee } \vee \text { teabag } \\
\text { steam } \wedge \text { teabag } \Rightarrow \text { hot_drink } &
\end{array}
$$

Fig. 6. Knowledge Base $\mathcal{A}$ for an espresso machine from [45].

\subsection{Decomposing KB for context-based reasoning and Harnessing}

The following example comes from [45] and it analyzes the behavior of an espresso machine whose functioning aspects are captured by a KB $\mathcal{A}$, whose axioms have been enumerated in Fig. 6 .

In order to work with Knowledge Harnessing some cases of [45] will be used. Authors obtain a partition of the KB according to the contexts induced by different use cases. The languages from these partitions can be used to compute conservative retractions, in order to check the completeness of the refined KB obtained in the above-mentioned paper. In [43] the corresponding conservative retractions are computed. In particular, for the context $\mathcal{A}_{1}$ induced by the variable set

$$
\mathcal{L}\left(\mathcal{A}_{1}\right)=\{\text { on_pump, ok_pump, water, man_fill }\}
$$

The conservative retraction $\left[\mathcal{A}, \mathcal{L}\left(\mathcal{A}_{1}\right)\right]$, is

$$
\left[\mathcal{A}, \mathcal{L}\left(\mathcal{A}_{1}\right)\right]=\left\{\begin{array}{l}
\text { ok_pump } \wedge \text { on_pump } \rightarrow \text { water, man_fill } \rightarrow \text { water }, \\
\text { man_fill } \rightarrow \neg \text { on_pump, } \neg \text { man_fill } \rightarrow \text { on_pump }
\end{array}\right\}
$$

Let us suppose that the system receives the formula when it is in contexts specified with the first language, $\mathcal{L}\left(\mathcal{A}_{1}\right)$

$$
F \equiv(\text { manfill } \leftrightarrow \text { water } \vee \text { on_pump }) \wedge \text { steam }
$$

In this case $K \forall_{\mathcal{L}\left(\mathcal{A}_{1}\right)} F$ because

$$
F_{\left\lceil\mathcal{L}\left(\mathcal{A}_{1}\right)\right.} \equiv \text { manfill } \leftrightarrow \text { water } \vee \text { on_pump }
$$

and $\left[\mathcal{A}, \mathcal{L}\left(\mathcal{A}_{1}\right)\right] \not \models F_{\left\lceil\mathcal{L}\left(\mathcal{A}_{1}\right)\right.}$ In this case, it holds that

$$
\omega_{\left(\mathcal{L}\left(\mathcal{A}_{1}\right)\right.}^{\mathcal{A}}(F)=2 / 5
$$

\section{Conclusions, related and future work}

In this paper the elements of a TWD model for knowledge harnessing have been presented, including the problem in this field of research. From the definition, of semantic nature, we have studied on the one hand the behavior before the extension of the language (which would be like the set of variables to take into account), and on the other hand, how to obtain the maximum useful information from an information which was incoherent with the knowledge base of the agent/system. The acceptance-rejection evaluation measures this feature, understanding that the number of models where it is validated (something like the number of possible contexts in the given language) quantifies the richness of that information.

The acceptance-reject evaluation estimates the conflict between the agent's $\mathrm{KB}$ and the new information, not just inconsistency. In fact the KB is not considered to be inconsistent. For this last case there are complete studies (see [46], where a systematic study/survey of the computational complexity of inconsistency measures is also presented ${ }^{1}$ ). In [47] an inconsistency measure is proposed based on a forgetting variable variant that could be used in this kind of situations.

Although the approach presented here is focused on the extension of the TWD paradigm in an agent/system, it is possible to extend it to the case of a multi-agent system following ideas similar to those of [48]. In addition, it would be possible to use this model to implement expert systems based on the knowledge of different experts such as [49] and for diagnosis (see [50]).

The use of variable forgetting in decision-making analysis is not completely new. However, this work is focused on its logical formalism for a particular problem. Language reduction is an important issue in a number of fields related with decision and reasoning. For instance, the equivalent problem within FCA would be attribute reduction (both in FCA and Fuzzy FCA [51]), in which decreasing the number of attributes used in reasoning tasks is important.

Reducing the number of attributes allows refining the knowledge and meaning of concepts obtained as a result of data processing [52]. It is known that the TWD represents an opportunity to establish or strengthen the relationships between

\footnotetext{
1 Recall that an inconsistency measure is a function $I: 2^{\text {Form }(\mathcal{L})} \rightarrow[0,+\infty]$ satisfying $I(K)=0$ if and only if $K$ is a consistent KB, whilst in the measures considered in this paper the 0 result denotes inconsistency.
} 
the FCA and the theories and techniques that are naturally incorporated within the TWD paradigm. There are proposals that combine FCA with TWD through three-way concept lattices (including duality [53]) and as a bridge between rough set concept analysis and FCA (see the recent [54]). In [55] authors use attribute reduction techniques on formal decision contexts in order to reduce the number of new concepts to be calculated. The same applies to cost-sensitive attribute reduction methods [56] and radius-based ones [57]. In general terms, these are approaches that aim optimizing the selection granularity [58]. Please note that reasoning on attribute dependence is, from a logical point of view, reasoning among these with implication logic, that is interpretable in propositional logic in turn for cases as FCA. Therefore KH could be applied by using more efficient forgetting operators [59]. See [60] for a general approach to the study of the dependence relationship.

In general terms, the problem of selecting (a minimal set) the most useful (e.g. for prediction) properties (features) is an important problem in a number of TWD models (see e.g. [61,62]). Considering different (informative) languages in knowledge harnessing would also allow adopting sequential or multi-class strategies to refine agent's decisions from its knowledge base. Using a framework for this kind of strategies (for agents [63], for multi-source information systems [64], multi-attribute group decision-making $[65,66]$ ) would allow estimating the information gain that could be obtained after increasing (or decreasing) the language to be Harnessed. Likewise, data dynamics need updating attributes and concepts [67].

In this paper we have analyzed and based the trisection on the conflicting knowledge, which once evaluated would be assimilated by the agent. Future work in this area will focus on effectively incorporating the notion of KH in different models of agency (especially in teleo-reactive architecture). Note that, as it was already mentioned, the assimilation of harnessed knowledge by the agent requires the extension of high-level behavior programs to maintain acceptance values in the actions that are decided. In particular, the triple-tower architecture [68] for Teleo-reactivity could be enriched in the so-called perception tower and model tower. This particular application will be the subject of future study.

The idea of estimating similarity (or conflict) proposed in this paper is related to other measures of defined similarity on other logics of limited expressivity (and therefore with models of relatively simple structure). For instance, in [69] authors define a Semantic Similarity Measure for DL-lite logic-based ontologies that work on $\mathcal{A}$-boxes via $\mathcal{A}$-box mapping. This measure could be useful for KH on DL-lite ontologies, considering the conflicting (but consistent) new information as an ontology itself in the sense that was commented in Sect. 1.4.1.

The defined evaluation also shares some similarity with a probabilistic approach to the case of incomplete reasoning. There are approaches in TWD to address the problem of dealing with incomplete information systems [70]. In fact, the presented approach could be reinterpreted in probabilistic terms (in a similar way they do in the cited paper), and it could be interesting to establish the properties of knowledge harnessing from that point of view. This question will be the subject of a future study.

\section{Declaration of competing interest}

We wish to confirm that there are no known conflicts of interest associated with this publication and there has been no significant financial support for this work that could have influenced its outcome.

\section{Acknowledgements}

We thank both reviewers and editors for their valuable comments and suggestions, which have improved significantly the content of this article.

\section{References}

[1] M.R. Genesereth, N.J. Nilsson, Logical Foundations of Artificial Intelligence, Morgan Kaufmann Publishers Inc., 1987.

[2] J. Lang, P. Liberatore, P. Marquis, Propositional independence - formula-variable independence and forgetting, J. Artif. Intell. Res. 18 (2003) $391-443$.

[3] F. Lin, R. Reiter, Forget it!, in: Proceedings of the AAAI Fall Symposium on Relevance, 1994, pp. 154-159.

[4] W.W. Bledsoe, L.M. Hines, Variable elimination and chaining in a resolution-based prover for inequalities, in: CADE, in: Lect. Notes Comp. Sci., vol. 87, Springer Berlin Heidelberg, 1980, pp. 70-87.

[5] Y. Moinard, Forgetting literals with varying propositional symbols, J. Log. Comput. 17 (5) (2007) 955-982.

[6] T. Eiter, K. Wang, Semantic forgetting in answer set programming, Artif. Intell. 172 (14) (2008) 1644-1672.

[7] J. Larrosa, E. Morancho, D. Niso, On the practical use of variable elimination in constraint optimization problems: 'still-life' as a case study, J. Artif. Intell. Res. 23 (2005) 421-440.

[8] Y. Zhang, Y. Zhou, Knowledge forgetting: properties and applications, Artif. Intell. 173 (16) (2009) 1525-1537.

[9] K. Su, A. Sattar, G. Lv, Y. Zhang, Variable forgetting in reasoning about knowledge, J. Artif. Intell. Res. 35 (2009) 677-716.

[10] G.A. Aranda-Corral, J. Borrego-Díaz, F. Gómez-Marín, Toward semantic mobile web 2.0 through multiagent systems, in: Agent and Multi-Agent Systems: Technologies and Applications, Springer Berlin Heidelberg, 2009, pp. 400-409.

[11] G.A. Aranda-Corral, J. Borrego-Díaz, J. Giráldez-Cru, Conceptual-based reasoning in mobile web 2.0 by means multiagent systems - knowledge engineering notes, in: ICAART 2012 - Proceedings of the 4th International Conference on Agents and Artificial Intelligence, Volume 2 - Agents, 2012, pp. 176-183.

[12] H.J. Levesque, R. Reiter, Y. Lespérance, F. Lin, R.B. Scherl, Golog: a logic programming language for dynamic domains, J. Log. Program. 31 (1) (1997) 59-83.

[13] G. De Giacomo, Y. Lespérance, H.J. Levesque, S. Sardina, IndiGolog: A High-Level Programming Language for Embedded Reasoning Agents, Springer US, 2009, pp. 31-72, Ch. 2.

[14] N.J. Nilsson, Teleo-reactive programs for agent control, J. Artif. Intell. Res. 1 (1) (1994) 139-158. 
[15] G. Aranda-Corral, J. Borrego-Díaz, J. Giráldez-Cru, Agent-mediated shared conceptualizations in tagging services, Multimed. Tools Appl. 65 (1) (2013) $5-28$.

[16] Z.W. Raś, A. Dardzińska, On reaching consensus by a group of collaborating agents, in: Flexible Query Answering Systems, Springer Berlin Heidelberg, 2009, pp. 583-592.

[17] P. Gärdenfors, Belief revision: an introduction, in: Cambridge Tracts in Theoretical Computer Science, Cambridge University Press, 1992, pp. 1-28, Ch. 1.

[18] M.V. Martinez, C. Molinaro, V.S. Subrahmanian, L. Amgoud, A General Framework for Reasoning on Inconsistency, Springer, 2013.

[19] L. Bertossi, Consistent query answering in databases, SIGMOD Rec. 35 (2) (2006) 68-76.

[20] M. Bienvenu, C. Bourgaux, F. Goasdoué, Computing and explaining query answers over inconsistent dl-lite knowledge bases, J. Artif. Intell. Res. 64 (1) (2019) 563-644.

[21] S. Jabbour, Y. Ma, B. Raddaoui, Handling disagreement in ontologies-based reasoning via argumentation, in: Web Information Systems Engineering WISE 2019, Springer International Publishing, 2019, pp. 389-406.

[22] P. Chatalic, G.H. Nguyen, M.C. Rousset, Reasoning with inconsistencies in propositional peer-to-peer inference systems, in: Proceedings of the 2006 Conference on ECAI 2006: 17th European Conference on Artificial Intelligence, IOS Press, 2006, pp. 352-356.

[23] R. Witte, N. Khamis, J. Rilling, Flexible ontology population from text: the owlexporter, in: International Conference on Language Resources and Evaluation (LREC), ELRA, 2010, pp. 3845-3850.

[24] P. Pu, L. Chen, User-involved preference elicitation for product search and recommender systems, AI Mag. 29 (4) (2008) 93.

[25] M. Schubert, A. Felfernig, F. Reinfrank, Reaction: personalized minimal repair adaptations for customer requests, in: Flexible Query Answering Systems, Springer Berlin Heidelberg, 2011, pp. 13-24.

[26] Y. Tzitzikas, C. Meghini, N. Spyratos, A unified interaction scheme for information sources, J. Intell. Inf. Syst. 26 (1) (2006) 75-93.

[27] A. Wakrime, S. Jabbour, On repairing queries in cloud computing, in: 2016 IEEE/ACS 13th International Conference of Computer Systems and Applications (AICCSA), IEEE Computer Society, 2016, pp. 1-6.

[28] A. Bidault, C. Froidevaux, B. Safar, Repairing queries in a mediator approach, in: Proceedings of the 14th European Conference on Artificial Intelligence, ECAI'00, IOS Press, 2000, pp. 406-410.

[29] S. Chlebowski, A. Gajda, Abductive question-answer system (AQAS) for classical propositional logic, in: Flexible Query Answering Systems, Springer International Publishing, 2017, pp. 3-14.

[30] K. Inoue, L. Wiese, Generalizing conjunctive queries for informative answers, in: Flexible Query Answering Systems, Springer Berlin Heidelberg, 2011, pp. $1-12$.

[31] Y. Yao, Three-way decisions with probabilistic rough sets, Inf. Sci. 180 (3) (2010) 341-353.

[32] Y. Yao, The superiority of three-way decisions in probabilistic rough set models, Inf. Sci. 181 (6) (2011) 1080-1096.

[33] Y. Yao, Three-way decision and granular computing, Int. J. Approx. Reason. 103 (2018) 107-123.

[34] Y. Yao, Three-way decisions and cognitive computing, Cogn. Comput. 8 (4) (2016) 543-554.

[35] Y. Yao, An outline of a theory of three-way decisions, in: Rough Sets and Current Trends in Computing, Springer Berlin Heidelberg, 2012, pp. 1-17.

[36] Y. Qian, X. Liang, Q. Wang, J. Liang, B. Liu, A. Skowron, Y. Yao, J. Ma, C. Dang, Local rough set: a solution to rough data analysis in big data, Int. J. Approx. Reason. 97 (2018) 38-63.

[37] M.K. Afridi, N. Azam, J. Yao, E. Alanazi, A three-way clustering approach for handling missing data using GTRS, Int. J. Approx. Reason. 98 (2018) 11-24.

[38] Y.T. Yan, Z.B. Wu, X.Q. Du, J. Chen, S. Zhao, Y.P. Zhang, A three-way decision ensemble method for imbalanced data oversampling, Int. J. Approx. Reason. 107 (2019) $1-16$.

[39] Y. Zhang, D. Miao, Z. Zhang, J. Xu, S. Luo, A three-way selective ensemble model for multi-label classification, Int. J. Approx. Reason. 103 (2018) $394-413$.

[40] T. Zhang, H. Li, M. Liu, M. Rong, Incremental concept-cognitive learning based on attribute topology, Int. J. Approx. Reason. 118 (2020) $173-189$.

[41] X. Jia, Z. Deng, F. Min, D. Liu, Three-way decisions based feature fusion for Chinese irony detection, Int. J. Approx. Reason. 113 (2019) $324-335$.

[42] Y. Zhang, D. Miao, J. Wang, Z. Zhang, A cost-sensitive three-way combination technique for ensemble learning in sentiment classification, Int. J. Approx. Reason. 105 (2019) 85-97.

[43] J.A. Alonso-Jiménez, G.A. Aranda-Corral, J. Borrego-Díaz, M.M. Fernández-Lebrón, M. Hidalgo-Doblado, A logic-algebraic tool for reasoning with knowledge-based systems, J. Log. Algebraic Methods Program. 101 (2018) 88-109.

[44] G.A. Aranda-Corral, J. Borrego-Díaz, M.M. Fernández-Lebrón, Conservative Retractions of Propositional Logic Theories by Means of Boolean Derivatives, Calculemus/MKM Lect. Notes Comput. Sci., vol. 5625, Springer, 2009, pp. 45-58.

[45] E. Amir, S. Mcllraith, Partition-based logical reasoning for first-order and propositional theories, Artif. Intell. 162 (1-2) (2005) 49-88.

[46] M. Thimm, J.P. Wallner, On the complexity of inconsistency measurement, Artif. Intell. 275 (2019) 411-456.

[47] P. Besnard, Forgetting-based inconsistency measure, in: Scalable Uncertainty Management, Springer International Publishing, 2016, pp. 331-337.

[48] X. Yang, J. Yao, Modelling multi-agent three-way decisions with decision-theoretic rough sets, Fundam. Inform. 115 (2-3) (2012) 157-171.

[49] A. Hernando, E. Roanes-Lozano, An algebraic model for implementing expert systems based on the knowledge of different experts, Math. Comput. Simul. 107 (2015) 92-107.

[50] J. Piury, L.M. Laita, E. Roanes-Lozano, A. Hernando, F.-J. Piury-Alonso, J.M. Gómez-Argüelles, L. Laita, A Gröbner bases-based rule based expert system for fibromyalgia diagnosis, Rev. R. Acad. Cienc. Exactas Fís. Nat., Ser. A Mat. 106 (2) (2012) 443-456.

[51] Y. Zhai, D. Li, Knowledge structure preserving fuzzy attribute reduction in fuzzy formal context, Int. J. Approx. Reason. 115 (2019) $209-220$.

[52] M. Wolski, A. Gomolińska, Data meaning and knowledge discovery: semantical aspects of information systems, Int. J. Approx. Reason. 119 (2020) 40-57.

[53] H. Zhi, J. Qi, T. Qian, L. Wei, Three-way dual concept analysis, Int. J. Approx. Reason. 114 (2019) 151-165.

[54] Y. Yao, Three-way granular computing, rough sets, and formal concept analysis, Int. J. Approx. Reason. 116 (2020) 106-125.

[55] J. Chen, J. Mi, B. Xie, Y. Lin, A fast attribute reduction method for large formal decision contexts, Int. J. Approx. Reason. 106 (2019) 1-17.

[56] Y. Fang, F. Min, Cost-sensitive approximate attribute reduction with three-way decisions, Int. J. Approx. Reason. 104 (2019) 148-165.

[57] Z. Jiang, K. Liu, X. Yang, H. Yu, H. Fujita, Y. Qian, Accelerator for supervised neighborhood based attribute reduction, Int. J. Approx. Reason. 119 (2020) $122-150$.

[58] Q. Wan, J. Li, L. Wei, T. Qian, Optimal granule level selection: a granule description accuracy viewpoint, Int. J. Approx. Reason. 116 (2020) 85-105.

[59] G.A. Aranda-Corral, J. Borrego-Díaz, J. Galán-Páez, A.T. Caballero, On Experimental Efficiency for Retraction Operator to Stem Basis, Springer International Publishing, 2019, pp. 73-79, Ch. 8.

[60] G. Chiaselotti, F. Infusino, Notions from rough set theory in a generalized dependency relation context, Int. J. Approx. Reason. 98 (2018) 25-61.

[61] A. Ferone, Feature selection based on composition of rough sets induced by feature granulation, Int. J. Approx. Reason. 101 (2018) $276-292$.

[62] M.S. Raza, U. Qamar, Feature selection using rough set-based direct dependency calculation by avoiding the positive region, Int. J. Approx. Reason. 92 (2018) 175-197.

[63] X. Yang, T. Li, H. Fujita, D. Liu, A sequential three-way approach to multi-class decision, Int. J. Approx. Reason. 104 (2019) 108-125.

[64] B. Sang, L. Yang, H. Chen, W. Xu, Y. Guo, Z. Yuan, Generalized multi-granulation double-quantitative decision-theoretic rough set of multi-source information system, Int. J. Approx. Reason. 115 (2019) 157-179. 
[65] B. Sun, W. Ma, B. Li, X. Li, Three-way decisions approach to multiple attribute group decision making with linguistic information-based decisiontheoretic rough fuzzy set, Int. J. Approx. Reason. 93 (2018) 424-442.

[66] J. Pang, X. Guan, J. Liang, B. Wang, P. Song, Multi-attribute group decision-making method based on multi-granulation weights and three-way decisions, Int. J. Approx. Reason. 117 (2020) 122-147.

[67] B. Long, W. Xu, X. Zhang, L. Yang, The dynamic update method of attribute-induced three-way granular concept in formal contexts, Int. J. Approx. Reason. (2020).

[68] N.J. Nilsson, Teleo-reactive programs and the triple-tower architecture, Electron. Trans. on Artif. Intell. 5 (2001) 99-110.

[69] H. Stuckenschmidt, A semantic similarity measure for ontology-based information, in: Flexible Query Answering Systems, Springer Berlin Heidelberg, 2009, pp. 406-417.

[70] X. Yang, H. Song, T.-J. Li, Decision making in incomplete information system based on decision-theoretic rough sets, in: Rough Sets and Knowledge Technology, Springer Berlin Heidelberg, 2011, pp. 495-503. 